\title{
Avancées dans les soins préhospitaliers d'urgence
}

\section{Jean Martin}

Dr med., membre de la rédaction

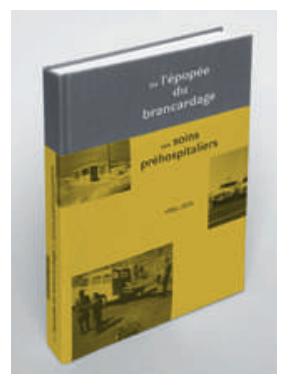

Georges Vittoz et coll. (dir. publ.) De l'épopée du brancardage aux soins préhospitaliers (1950-2015)

Lausanne: Fondation Urgences Santé; $2016 .{ }^{1}$ 287 pages. $50 \mathrm{CHF}$. ISBN 978-2-8399-1895-4
1 Peut être obtenu auprès de Fondation Urgences Santé, Rue Dr César-Roux 31 , 1005 Lausanne.
Ce livre a d'abord pour moi une résonance personnelle, en présentant l'évolution intervenue dans les secours d'urgence aux malades et blessés (sur les routes, à leur domicile, à leur travail) dans le canton de Vaud au cours des soixante dernières années, sous l'égide du Service de la santé publique; service dont j'ai été membre de 1976 à 2003. Sans avoir été principalement en charge du domaine en question, j'en ai suivi le développement - tout en participant, à une époque qu'on pourrait presque dire héroïque (fin des années 1970), à la formation de base de ceux qui œuvraient comme ambulanciers.

Alors que, trop souvent, on peut regretter que l'histoire de la santé publique ne soit pas adéquatement documentée, un tel ouvrage répond à cette crainte pour ce qui est devenu la chaîne des urgences. Quatre parties: 1) Introduction; 2) Evolution de 1950 à 1980; 3) Une nouvelle ère des urgences préhospitalières, 1981 à 2015; 4) Lorsque la petite histoire rejoint la grande. La troisième partie est la plus importante, retraçant les développements aux plans historique, politique, administratif, technique, de la formation et de la planification. La quatrième consiste en une collection de témoignages-vignettes avec les souvenirs et appréciations d'une vingtaine d'acteurs (ambulanciers, médecins, responsables hospitaliers et de services publics, chef de la base lausannoise de la Rega/ Gass). On relèvera, selon les termes du médecin cantonal Karim Boubaker, que la responsabilité de l'ouvrage a été confiée «à la personne qui a le plus œuvré au sein des services de l'Etat pour que chaque citoyen puisse bénéficier de la prise en charge la plus efficiente possible, Georges Vittoz».

On y trouve de nombreuses illustrations, documents des services publics et d'autres organisations, correspondances, éléments concernant les formations offertes, grilles d'évaluation des patients, tables montrant l'évolution du nombre d'interventions, photos. Dans l'avantpropos de Vincent Barras, directeur de l'Institut lausannois d'histoire de la médecine: «Le sentiment de l'urgence guide la médecine depuis toujours. La nécessité de venir en aide, toutes affaires cessantes, à la personne en difficulté vitale s'est doublée de pratiques médico-sanitaires plus ou moins organisées. [Ici comme ailleurs,] mêmes hésitations et résistances, et tout à la fois même enthousiasme de quelques personnalités soucieuses du bien public [...] La mise en place décrite ici répond aussi à une nouveauté, l'émergence d'une société urbanisée, de plus en plus conditionnée par les installations techniques - alors qu'un éventail de situations inédites se déploie en quelques décennies (explosion démographique, risques industriels, accroissement du trafic mécanisé). Du coup, le récit de cette histoire ressemble à une épopée.»

Entre autres est décrite l'évolution du rôle de l'Etat, qui au cours des décennies élargit et coordonne les initiatives prises au départ par d'autres (garagistes privés, ville de Lausanne, Rega pour les hélicoptères par exemple). Progressivement, y compris par le biais d'interventions et décisions parlementaires, l'autorité publique cantonale assume un leadership au plan de l'organisation et du soutien financier. L'élargissement des compétences des ambulanciers, instauré parallèlement à la médicalisation des interventions, constitue une évolution marquante.

\section{Une politique qui associe l'Etat et tous ses} partenaires. Dans ce système, personne n'a le pouvoir mais tout le monde profite du résultat.

Selon les auteurs: «Cette rétrospective est destinée à un large public. Elle doit également permettre aux acteurs actuels de mieux cerner les raisons des différents choix retenus et de mesurer les difficultés rencontrées.» Dans leur conclusion: Le développement des urgences préhospitalières a été «réalisé par étapes avec un cap: la chaîne des urgences. Ceci par une politique transparente dans ses objectifs et ses coûts, dont les objectifs successifs sont confrontés à l'épreuve des faits. C'est surtout une politique qui associe étroitement l'Etat et tous ses partenaires. Dans ce système, personne n'a le pouvoir mais tout le monde tire profit du résultat.» C'est bien là une manière de décrire comment le plus souvent, dans notre pays, évoluent le système de santé et ses diverses composantes. 